\title{
Closed-Loop Manufacturing for Aerospace Industry: an integrated PLM-MOM solution to support the wing box assembly process
}

\author{
Melissa Demartini ${ }^{1}$, Federico Galluccio ${ }^{2}$, Paolo Mattis ${ }^{2}$, Islam Abusohyon ${ }^{1}$, Raf- \\ faello Lepratti ${ }^{3}$, Flavio Tonelli ${ }^{1}$ \\ ${ }^{1}$ DIME - Department of Mechanical Engineering, Energetics, Management and Transportation, \\ Polytechnic School, University of Genoa, ITALY \\ ${ }^{3}$ Siemens Italy S.p.A., Via Enrico Melen 83, 16152, Genoa, ITALY \\ ${ }^{2}$ Siemens AG., Gleiwitzerstr. 555, 90475 Nuremberg, GERMANY
}

melissa.demartini@dime.unige.it

\begin{abstract}
.
The aim of this research is to provide an example of the importance that integrated Product Lifecycle Management (PLM) and Manufacturing Operation Management (MOM) systems have in realizing the Digital Manufacturing. The research first examines what the Digital Manufacturing involves and then identifies Digital Twin and the related Digital Thread as key elements. PLM and MOM solutions support the Digital Twin and the Digital Thread allowing the exchange of product-related information between the digital manufacturing model and the physical manufacturing execution. A Digital Twin of a wing box and its assembly process is created in PLM by building the bill of material and bill of process. Then it is shown how in MOM system the production phase is facilitated by managing production operations, advanced scheduling and supporting the execution of the processes and how the analysis of the manufacturing performance is possible. The result integrating these systems is to have the right information at the right place at the right time along with the related benefits in terms of costs, time and quality. The activity has been developed in Siemens Industry Software under the European Project AirGreen 2, an integrated research action of the REG IADP (Regional Innovative Aircraft Demonstration Platform) part of the Joint Technical Programme, the steering and coordination of LEONARDO Aircraft. The AirGreen 2 project is an Innovation Action funded by the Clean Sky 2 Joint Undertaking under the European Union's Horizon 2020 research and innovation programme, under Grant Agreement Nº07089 REG IADP).
\end{abstract}

Keywords: Digital manufacturing, aerospace industry, closed loop manufacturing.

\section{Introduction}

The digital transformation has already changed the way products are designed, produced and delivered and this has a profound impact on manufacturing companies [1] 
[2]. In this competitive scenario, manufacturing companies perform by using up-to-date information to progressively remove delays to the management and execution of its critical business processes. Therefore, they need to monitor their value-creation processes controlling both the internal (e.g. scheduling flexibility) and external events (e.g. customization and volume flexibility) in order to maintain their competitive advantage [1]. In the light of the above, the success of a manufacturing company depends upon effective coordination across all stages in a product's development, from design and production up to aftermarket services [3]. Companies should define and optimize manufacturing processes, manage data and encourage collaboration between different types of engineers by incorporating both digital and product definitions. Thus, an integrated system is necessary to manage the different production stages and comprising various business functions. To this end, the aim of this paper is to introduce an innovative system to bridge the vertical integration gap between the engineering and manufacturing domains leading to an integrated production management system, which includes Product Lifecycle Management (PLM) and Manufacturing Operation Management (MOM). Thanks to the advancement of innovative technologies, such as Digital Twin and a Digital Thread, this gap could be enclosed. Through digital twin, any entity or physical system, product or process can be represented in a simulated way to respond immediately to external changes to prevent problems solving them in real time or improving performances. The digital twin is fed by information from the digital thread [6], which is the chain of information that connects all the involved parties with data to design and produce products [7]. The term "thread" is used because it interweaves and brings together data from all stages of the product and production lifecycles [8]. Bringing together the digital twin and thread, it is possible to create a collaborative, connected information loop, the so - called closed loop manufacturing (CLM). CLM enables companies to synchronize and optimize production across product design, production planning, manufacturing execution, automation and intelligence from consumer use in the field.

\subsection{Research Goals and Questions}

The purpose of this research is the design, implementation and industrial evaluation of an innovative system to bridge the vertical integration gap between the engineering and manufacturing domains. A digital twin of a wing box and its assembly process is created in PLM by building the bill of material (BOM) and bill of process (BOP). Then it is shown how in MOM system the production phase is facilitated by managing production operations and supporting the execution of the processes leading to a CLM. The literature is witnessing of the transition of the aerospace industries, which are moving from fixed to flexible production cells, requiring a higher level of vertical integration between enterprise systems and shop floor controls and managing a higher level of customization. Aerospace companies are deploying new digital technologies in manufacturing to speed up production and reduce costs. In essence, this study is conducted to answer the following research question: 
RQ: A solution based only on ERP system can properly manage highly complex product's BOMs?

When the products are highly complex, the integration between PLM-MOM is necessary to manage BOMs that otherwise can't be managed using only the ERP. The BOM involves unique challenges for the management of information across business functions. It is initially created by engineering to meet engineering needs, but multiple departments rely on it and need to use the information it contains in different ways. Managing these different views, integrating the various engineering disciplines, and maintaining completeness and traceability become more difficult as product complexity increases. To answer this research question, this study provides a qualitative literature review to analyze the current scenario and then a proof of concept of an integrated PLM and MOM system to demonstrate its benefits. This paper is organized as follows: in Section 2 the literature review is depicted, Section 3 describes the methodology adopted for this paper. Section 4 presents the results, while in Section 5, conclusions are described.

\section{Research Background}

The literature review has been performed by selecting papers from Scopus. Authors chose this database for its ample coverage of articles in this field [9]. The Authors' strategy was to identify articles that included "Smart manufacturing", "Product lifecycle" and "Digital twin" as keywords in all fields. Additionally, the Authors considered various synonyms of each of these terms such as "smart factory" and "Digital thread". The aforementioned search technique allowed identification of 38 academic papers, which were rigorously reviewed in order to evaluate their adherence to the study. After reading all the papers, none of them talks about the importance of using the digital twin to realize the interconnection and the interaction between the PLM and MOM platforms within the manufacturing enterprises. This gap in the literature review shows that more work is needed in this area. Today's manufacturing enterprises use platforms to manage the product design as well as the production planning and the manufacturing execution. The design and implementation models are developed on a PLM platform. Modern PLM systems bring together product and process development, allowing interaction in the development process. Instead, the MOM platform covers the scheduling and execution of the work orders based on the development process. Usually PLM and MOM platforms do not have an appropriate interaction with each other and therefore the world of product and process design and that one of production and execution are not integrated for flexible, scalable production processes that maximize the responsiveness to real-time manufacturing events. Building a CLM is the solution to solve this problem. In this research, in order to achieve CLM and support the journey to Industry 4.0, PLM and MOM platforms have been made able to communicate with each other. It means they have access to, and understand the language of the others. MOM is where the virtual plan becomes the physical reality and it is also where decentralized production can be globally orchestrated. To conclude, it is possible to claim that the topic of the digital manufacturing starts to be studied deeper over the last year. Nevertheless, it must 
be said the fact that over 38 articles identified, only a few were interesting could mean a lack of knowledge of the importance of the CLM from the scientific literature point of view. This lack represents the starting point of this paper, that try to fill this gap by showing an application of the integration between PLM and MOM systems in the aerospace industry.

\section{$3 \quad$ Methodology}

A proof of concept software based on PLM and MOM platform has been implemented, the activity has been developed in Siemens Industry Software under the European Project AirGreen 2, an integrated research action of the REG IADP (Regional Innovative Aircraft Demonstration Platform) through the steering and coordination of LEONARDO Aircraft. The project aims to develop and demonstrate innovative concepts and methodologies enabling the realization of a wing of new generation. This wing will be characterized by an innovative structure, as a result of an improved life cycle design; a high level of adaptability, enabling load control and alleviation strategies, and enhancing the aerodynamic performance at the different flight regimes; an innovative aerodynamic design, oriented to the preservation of the natural laminar flow and for the drag reduction. Among the various work packages that have been set in order to achieve these goals, Siemens Industry Software has been involved in that one called "Innovative Wing Structure D\&M (Design \& Manufacturing)". The "Innovative Wing Structure D\&M" scope is the development, verification and optimization of suitable advanced technologies and materials for the manufacturing and assembly of the wing box aimed to the reduction of the manufacturing costs and the improvement of the environmental aspects if compared to a traditional process. In this context, Siemens and Leonardo Aircraft contribute to the development of a process for the industrial case study under the European Project AirGreen 2 - task 2.1.1.15 "Wing Box Assembly Manufacturing Executing System (MES) in a Manual and Automatic assembly environment collecting Manufacturing Data and Performances KPIs.

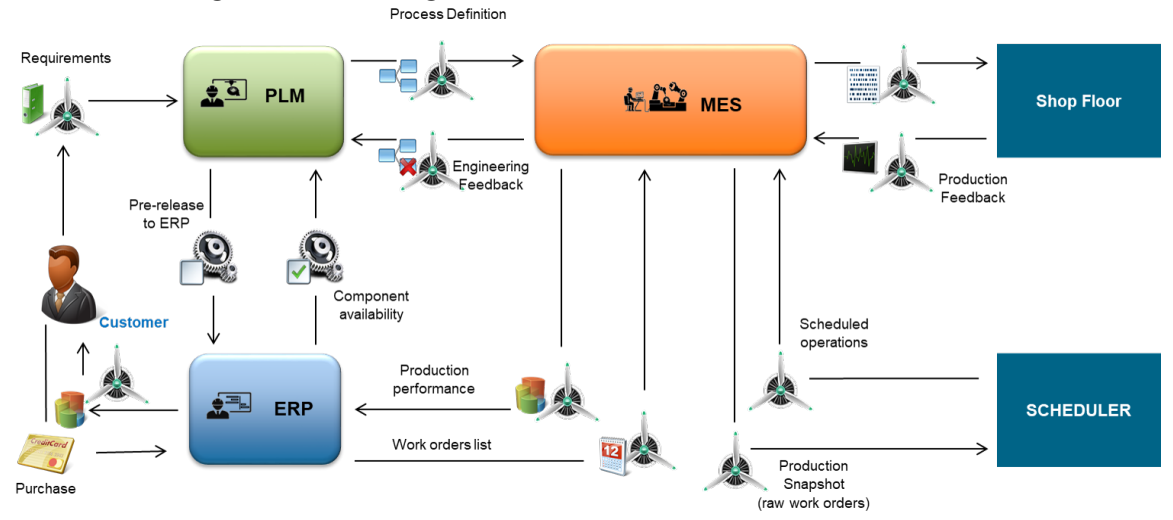

Figure 1: Architecture of the CLM

In the industrial case analyzed during this research, a wing box assembly method based on a predictive Dimensional Management (DM) strategy has been used. It involves the 
reverse engineering of some main wing components into a Digital Twin, representative of the actual shape of the parts within a defined level of accuracy. Intermediate parts are then defined into the Digital Twin Environment and transformed into real items through a dedicated manufacturing method developed by Leonardo Aircraft. The achievement of quality requirements is ensured adopting a Verification and Validation approach during the Product Life Cycle developmental stages. Customer requirement, Process Capability, Tooling and Methods are developed in concurrent engineering with the design within the Digital twin environment with the aim to guarantee customer needs expressed in terms of geometrical requirements at each product level (KCs), with a top down approach. Both RSS Stack-up evaluations and Montecarlo simulations are used to support variation propagation analysis and tolerance allocation in 1D and 3D; the Build-Up strategy achieved by extensive use of DM allows a set of advantages: i) readiness for the digital factory revolution since all feedback from shop floor is fully implemented; ii) allows lower fixed costs and easier design changes; iii) reduce labor costs and iv) involves less assembly variability. Despite all the benefits of Build-Up, there are several risks associated with it. Primarily, it requires much more attention to issues of variation. For this reason, greater attention shall be paid to enforce Statistical Process Control strategies including proper feedback and reporting in the Digital Twin trough the PLM applications. Therefore, in addition to the process based on the BuildUp strategy an alternative assembly method has been developed in order to mitigate potential failures discovered or predicted. It is a Back-up solution, based on failure scenarios analysis and tolerances (defective parts).Both processes have been modeled on the PLM platform but only the first one has been transferred to the MES.

\section{$4 \quad$ Results and Discussions}

In the Siemens PLM solution (Teamcenter) the BOM of the wing box as well as the BOPs related both to the machining process and to the wing box assembly have been built. They are the virtual twin of the product and virtual twin of the processes, respectively, which will be maintained in one shared manufacturing master data model and authored in one environment for production planning and production execution. BOPs have been built by creating process structure and creating operations. The link between the product structure and process is formed by associating the end item of the $\mathrm{BOM}$ as target for the BOP structure. Parts from the BOM are then assigned to the operations as "consumed parts". BOPs also define the data that must be collected during each operations and the electronic work instructions (EWI) that allow the communication of all the manufacturing process information between engineers and operators. The two assembly methods have been implemented in the PLM using a $150 \%$ BOP. With a $150 \%$ BOP is associated a $150 \% \mathrm{BOM}$, they are just other names for variant structures, or more specifically, configurable structures. Configurable BOMs and related BOPs have one or more optional or different components and/or optional or different operations, which, when properly set, define a specific variation of a product and its production. A "Configurable BOM" alias " $150 \%$ BOM" alias "Variant BOM", is used by manufacturer when dealing with highly complex product such as in the aerospace domain and 
when there is a need to maintain a balance between configurability, time to manufacture, and preserving the cost under threshold. A configurable bill of material contains all the parts that are required to manufacture the material to a customer's specific requirements. A "Select-Condition" is then applied on this predefined structure that determines whether a part is to be included in the final BOM or not. Modern PLM tools enables manufacturing companies to better adapt to engineering practices and facilitates efficient management of these configurable BOMs using a unit effectivity method. Effectivity might be defined as a date, serial number or, in a more complex way, as a "unit". Effectivity definition originally comes from ERP environment. The most typical example is "date effectivity" which defines the available to a particular part or item. PLM originally was created without effectivity in mind. Most of engineering systems were "revision" oriented rather than "effectivity" oriented. It means PLM is used to manage different revision of objects rather than defines their effectivities and it is the ERP system that manage the BOMs and their variations. A 150\% BOM haven't been built but the same BOM without any variation can be composed by different parts depending on the adopted assembly method. Custom parts based on Digital Twin configuration will therefore be different from those used in the other assembly method that involves traditionally fettled and drilled parts. Once the BOMs, the BOP and the related resources have been verified, the components of the $150 \%$ (master data like e.g. Material, Process, Operation, etc.) have been released from the PLM to the MES Siemens solution. Structures, relations and configurations are not relevant in this step. Instead, it only cares about the building blocks of the unconfigured structures. Then, imagining that a request to produce a wing box using the DA assembly method comes from an ERP system, in the MES work orders have been created specifying the effectivity needed and the download of the configured production structures (100 \% BOPs) from the PLM is then automatically triggered. Therefore, in the manufacturing execution system several work orders related to the just released processes has been executed. During their execution, materials are displayed as information about what has "to be consumed" for each single operation or step with the aim to guide the operators in consuming the right parts at the right places (operation station), at the right time (when the product is in the operation station) and collect in real-time the information about their consumption. Data collection form containing all the data and measures to be collected are displayed in the operator environment as well as the supporting documents and the work instruction to perform the operations, so that in a single place operator can find what to do and what to measure. Both work instruction and data collection form directly come from PLM model as part of the BOP: this means the MES shows, operation by operation, the document version according to the most updated BOP. Once the processes have been executed on the MES, the manufacturing operations performance are analyzed. The reporting and analysis layer have the scope to provide transparency to an information layer higher than the operational, which is supported by the as build and genealogy of the product and work orders inside the MES. It is the layer where we can compare work orders and get relevant KPIs and information about products, materials, defects and equipment outside the boundaries of a specific work order. It supports decision makers in the analysis of the plant information giving them the opportunity to understand how eventually modify and improve the products or its related process. The 
digital model of the production and process has been continuously compared with actual production to reduce the differences between as planned and as build. A PLMderived and MES-driven BOP can ensure that every material and operation is performed as it was intended. Requirements; which has been established in the earliest phases of engineering, become features of the aircraft design, dimensioned in the 3D models, published in the BOP, send to operators in EWIs, have been measured and finally stored in the as-built thanks to the digital thread. The as-built record can be compared to the as-ordered configuration to highlight deviations on the shop floor versus customer expectations. Once published back to the Digital Twin it can be matched against the as-engineered definition. The Build-Up strategy analyzed in this research is possible since it integrates the information available from production databases and methods into the design. This means that time is not spent working on components within the final assembly where a high level of capital expenditure is then tied up in these operations and a bottleneck to production exists. Finally, to answer the $R Q: A n$ ERP system can properly manage highly complex product's BOMs?

When the products are highly complex, the integration between PLM-MOM is necessary in order to manage their BOMs that otherwise can't be managed using only the ERP. PLM allows managing and maintaining both the engineering and the manufacturing aspects of a BOM in a single context during each stage of a product development process. The BOM is the result of a lot of design activity and if an ERP system, coming in at the end of the process, hold it we might fail to represent the real way in which the decisions, the logic, the engineering rules are built in to the product. ERP solutions generally do not actually optimize or have development tools for defining what the manufacturing BOM is. They just focus on executing a defined BOM. Modern PLM systems instead have the tools to make changes, analyze and optimize the BOM and therefore it makes sense to manage it through these solutions. To conclude, the problem of "siloed data" can be largely eliminated, this because there is now a bi-directional level of communication between the ERP, PLM, business systems and the shop floor, allowing for greater and immediate control of the business on the production floor.

\section{Conclusion}

Among all industries aerospace sector has been identified as an excellence case to show the benefits that Digital Twin, Digital Thread and CLM managed through PLM and MOM solutions, can bring. As demand for aircraft continues to grow, the aerospace industry has one of its largest opportunities to drive down existing backlogs, and increase competitiveness moving forward in integrating the world of product and process design and that one of production and execution into one common manufacturing model. Aerospace and systems will be built in a far more complex manner. Newer technologies and processes require new methods of manufacturing. In order to face these challenges, aerospace manufactures should introduce integrated PLM and MOM solutions into their manufacturing processes and thus obtain optimized order management, an automated advanced planning and scheduling for coordination of the supply chain flows and optimization of material synchronization, and all the benefits deriving from 
a CLM. In this regard, using the combined power of both PLM and MOM technology is a key part of company digitalization process. To drive innovation, it is essential to have the right technology in place in order to reduce development time and deliver high-quality solutions, with the ability to adapt to changes easily at every stage of the process.

\section{References}

1. Burger, N., Demartini, M., Tonelli, F., Bodendorf, F., Testa, C. Investigating Flexibility as a Performance Dimension of a Manufacturing Value Modeling Methodology (MVMM): A Framework for Identifying Flexibility Types in Manufacturing Systems (2017) Procedia CIRP, 63, pp. 33-38.

2. Diana C, Ioan P. Industrie 4.0 by Siemens: Steps made Next. Journal of Cases on Information Technology; 2018

3. Fei T, Jiangfeng G, Qinglin Q, Meng Z. Digital twin-driven product design manufacturing and service with big data. International Journal of Advanced Manufacturing Technology; 2017.

4. Fei T, Meng Z. Digital twin shop-floor: A new shop-floor paradigm towards smart manufacturing; 2017.

5. Giuseppe A, Marco S, Paolo P. Anetworked production system to implement virtual enterprise and product lifecycle information loops. 2017.

6. Demartini, M., Tonelli, F., Damiani, L., Revetria, R., Cassettari, L. Digitalization of manufacturing execution systems: The core technology for realizing future smart factories (2017) Proceedings of the Summer School Francesco Turco, 2017-September, pp. 326-333.

7. Mike P, Andreas A, Donald F, Victor M, Marcus H. Smart connected digital factories: Unleaching the power of Industry 4.0. Cloud computing and services science selected papers; 2018.

8. Haijan Z, Guohui Z, Qiong Y. Digital twin-driven cyber physical production systems towards smart shop-floor. Journal of Ambient Intelligence and Humanized Computing; 2018.

9. Demartini, M., Tonelli, F. Quality management in the industry 4.0 era (2018) Proceedings of the Summer School Francesco Turco, 2018-September, pp. 8-14. 